\title{
The relation between comprehending and remembering some complex sentences
}

\author{
MARCEL ADAM JUST and PATRICIA A. CARPENTER \\ Carnegie-Mellon University, Pittsburgh, Pennsylvania 15213
}

\begin{abstract}
This research concerns the distinction between processes in sentence comprehension and those in sentence memory. Comprehension was monitored by timing subjects while they decided whether a sentence was true or false of their knowledge of the world. The memory process was tapped by examining subjects' incidental memory for the sentences they had previously verified. The verification latencies indicated that at the time of comprehension, sentences like It is true that a fire isn't cold were often recoded into an equivalent affirmative form, namely, It is true that a fire is hot. However, negative sentences like It isn't true that a fire is cold were not recoded during comprehension. Recoding in recall occurred only for those types of sentences that were recoded at the time of comprehension.
\end{abstract}

Performance in a typical sentence-recall study is the culmination of many stages of processing, beginning with the initial interpretation of a sentence, the storage of the interpreted information, and finally the retrieval of the stored information. If the memory performance bears any interesting relation to the stimulus conditions, it is generally not possible to determine which of the processing stages is. responsible for the relation. In order to make such localization possible, it is necessary to have an account of the processing history of the information as it passes from stage to stage. This paper traces the processing history of two kinds of embedded negative sentences to show how differences in the initial comprehension may account for the patterns of sentence recall.

The difficulties in drawing inferences from memory performance have been compared to solving a single equation with two unknowns, namely, comprehension and memory (Fillenbaum, 1973). Comprehension processes. such as those influenced by the extralinguistic context of the communication or the linguistic structure of the sentence, can certainly affect how a sentence is remembered. Alternatively, subsequent memorial processes, such as transfer into long-term memory, forgetting from long-term memory, or reconstructive processes at time of recall. could affect memory performance. As an example of how memory data entail two unknowns, we can consider Fillenbaum's (1966) own influential study of memory for aftirmative and negative sentences. He

\footnotetext{
The order of authors is arbitrary. This paper represents a collaborative effort. Requests for reprints should be addressed to Marcel Adam Just. Psychology Department, Carnegie-Mellon University, Pittsburgh, Pennsylvania 15213. We thank Joe Christie, Dave Kieras, and Bill Leaf for their constructive help in various phases of this project. The research was partially supported by National Institute of Education Grant NIE-G-74-0016 and National Institute of Mental Health Grant MH-07722.
}

found that negative sentences like The door isn't open are sometimes recalled in a recoded form like The door is closed. But as Fillenbaum correctly pointed out, the results could be attributed to processes occurring either at the time the sentences were comprehended or at the time of retrieval. Solving an equation with two unknowns depends crucially upon obtaining two independent estimates of the unknowns. In order to assess the relative contributions of comprehension and memory to recall performance, it is necessary to determine how a sentence is represented during comprehension. independently of the memorial data.

To determine how a sentence is internally represented and processed during comprehension, one can examine certain aspects of performance during a comprehension task. The comprehension task used here required people to read a sentence and verify whether it was true or false based on their knowledge of the world, all while being timed. The verification task provides a way of monitoring the comprehension processes during the initial sentence presentation-a necessary condition for unconfounding comprehension and memorial effects. In addition, a verification task assures that each sentence is comprehended at least to the level at which its truth value can be extracted. The response latencies in sentence verification can be analyzed in terms of a processing model proposed previously (Carpenter \& Just, 1975). The model allows one to infer how certain kinds of complex sentences are represented and processed at the time of comprehension. Then it is possible to examine how the comprehension processes relate to subsequent memory performance.

The particular sentences used in the current experiment included the superordinate clause $I t$ is true that... (e.g., It is true that a fire is hot). It was assumed that sentential information might be represented propositionally in these tasks (cf. Clark \& 
Chase. 1972: Kintsch. 1972). In this propositional notation. words denote abstract entities that are not necessarily linguistic. The representation of the affirmative sentence may be (AFF. (HOT. FIRE)). meaning heat is predicated of fire and the predication is affirmative. Such a sentence can be negated in two ways. With one type of negation. the negative has a small scope, namely the inner predication: It is true that a fire isn't hot. This will be called predicate negation. (The scope of a negative is simply the range of constituents to which the negative applies.) The representation of this sentence is postulated to be (NEG. (HOT, FIRE)). The second type of negation has a larger scope, since the negative is in the superordinate clause, where it applies to the entire inner proposition: It isn't true that a fire is hot. This type of negation will be called denial. The embedding clause reverses the truth value of the embedded clause. Such a denial may be represented as (NEG, (AFF. (HOT, FIRE))). These representations are consistent with prior linguistic analyses (Jackendoff, 1969; Klima. 1964) as well as response latency analyses (Carpenter \& Just. 1975).

Previous research indicates that these two types of negatives are processed differently during comprehension (Carpenter \& Just, 1975). Predicate negatives are sometimes recoded into an equivalent affirmative form during the verification task. For example, It is true that a fire isn't cold may be recoded into It is true that a fire is hot before it is compared to the subject's knowledge of fires. This inference was made because the latencies for predicate negatives resembled those for affirmative sentences in that the true case was verified faster than the false case. The same pattern (of true negatives being verified faster than false ones) is also obtained when subjects are explicitly instructed to recode negatives into equivalent affirmative form (Carpenter, 1973; Trabasso, 1972; Wason, 1961; Young \& C hase, Note 1). Unlike predicate negatives, denials are kept in their regular, unrecoded form during verification. This inference is based on the result that false denials are verified faster than the true ones. In addition, the overall verification time was found to increase from affirmatives to predicate negatives to denials.

A precise model of how the sentence is compared to the picture can explain how people verify such affirmative and negative sentences (Carpenter \& Just, 1975). The model can also be used to infer whether or not subjects recode the predicate negatives. Specifically, if the true predicate negatives are verified faster than the false ones, then one can infer that the predicate negatives are being recoded. One can also examine whether predicate negatives tend to be recalled in a recoded form more often than denials. If so, the result would establish a strong relationship between the comprehension and recall of these negative sentences.

\section{METHOD}

\section{Materials}

Fasch person was presented with 60 different sentences. consisting of 10 exemplars of each of the six information conditions. Examples of the six information conditions are: True affirmative-lits true that a fire is hot: false affirmative-lis true that a wrestler is weak: tsue predicate negative-lis true that a villain isn't kind: false predicate negative-lt's true that an elephant isn't large: true denial-It isn' true that a rocket is slow: talse denial-It isn't true that a midget is short.

These sentences were constructed with 60 different concrete nouns (e.g. fire and freezer) and 30 pairs of adjectives (e.g.. hot and $(w /()$. No pretixed adjectives such as unhuppy were used. A subject saw each noun and each adjective only once. Each of six different groups of subjects saw the same noun-adjective combunation in a different information condition. The 60 different noun-adjective pairs were divided into six blocks of 10 pairs each. These six blocks were then combined with the six information conditions and the six groups of subjects in a Latin square design.

In addition to the 60 test sentences. there were 18 more buffer sentences, consisting of three exemplars of each of the six information conditions. Nine of the buffer sentences preceded the test sentences. and the other nine followed. These buffer sentences were designed to serve as practice trials in the verification task. and to attenuate any recency and primacy effects in the memory for the test sentences. The buffer sentences were not included in the analisis.

The order of the 60 noun-adjective pairs was initially randomized and then kept the same for every group of subjects. Subjects were assigned to one of the six groups randomly. with three subjects per group.

\section{Procedure}

The buffer and experimental sentences were presented in a verification task. The subject initiated a trial by pressing a microswitch. Half a second later. the sentence appeared in the tachistoscope. The subject was timed while he read the sentence and responded true or false by pressing one of two response buttons. All subjects responded true with their dominant hand. The sentences were typed in elite type and exposed in a tach istoscope at a distance of $58 \mathrm{~cm}$ subtending no more than $8 \mathrm{deg}$ of visual angle. The entire veritication task took about $10 \mathrm{~min}$.

Shortly after the end of the verification task, subjects were given a recall task to test their incidental memory for the sentences. The incidental memory task eliminates the possibility of subjects employing unusual memorizational strategies during the sentence presentation.

The subject was given a list of 60 noun prompts taken from the 60 experimental sentences. The nouns were presented in sentence frames like $I t$ is ___true that a fire is t__ and were listed in the same order as the parent sentences had been presented in the veritication task. Subjects were told to recall the adjective and, if there had been a negation, to insert it in the appropriate position. They were encouraged to recall the complete sentence accurately, but to give partial recall if they could not remember all of it. The recall task was self-paced, but the subjects were told to finish within $20 \mathrm{~min}$. The 18 paid subjects in the experiment were local college students.

\section{RESULTS AND DISCUSSION}

\section{Verification Time}

Latencies for incorrect responses $\mathbf{8 . 3 \%}$ were replaced by estimated latencies, assuming additivity of the effects of the independent variables (Winer, 1971). The result of central interest is how the verification latencies for the two kinds of negation were affected by truth value. Overall, predicate 
Table 1

Mean Verification Time in Milliseconds \pm Standard Error (and Percentage Error) for the Six Conditions

\begin{tabular}{|c|c|c|c|}
\hline $\begin{array}{l}\text { Stimulus } \\
\text { Sentence }\end{array}$ & True Case & False Case & Mean \\
\hline Affirmative & $2745 \pm 146 \quad(4 \%)$ & $2964 \pm 170(0 \%)$ & 2854 \\
\hline $\begin{array}{l}\text { Predicate } \\
\text { Negative }\end{array}$ & $3677 \pm 201 \quad(12 \%)$ & $3747 \pm 216(6 \%)$ & 3712 \\
\hline Denial & $4367 \pm 269 \quad(15 \%)$ & $3872 \pm 187(13 \%)$ & 4120 \\
\hline
\end{tabular}

negatives were verified slightly faster when true; by contrast, denials were verified much faster when false, as shown in Table 1. This interaction is statistically significant, $F^{\prime}(1,144)=4.92, p<.05$. (Because of the two random factors, people and propositions, the ratio of mean squares is tested by a quasi-F ratio, denoted, $F^{\prime}$, cf. Clark, 1973.) Since predicate negatives were verified faster when true, we can infer that they were often recoded into an equivalent affirmative form. However, denials retained their negative format.

Although the true predicate negatives were verified faster than the false ones, the absolute difference between true and false latencies for predicate negatives was smaller than for affirmatives or denials. One possible reason for the smaller true-false difference is that predicate negatives were recoded less than $100 \%$ of the time.

Previous research has also shown that latencies tend to increase linearly from the affirmative, to the predicate negative, to the denial case. The increase was monotonic but not linear in this experiment; the latencies for predicate negatives were greater than expected. The extra time taken for predicate negatives may possibly have been consumed in retrieving from semantic memory the antonym of the adjective presented in the sentence. In the previous studies involving recoding (Carpenter \& Just, 1975; Just \& Carpenter, 1975), there were generally only two adjectives in the entire experiment. So the time required to retrieve the antonym may have been relatively short, since one of only two symbols was always being retrieved during the recoding trials. By contrast, the predicates in each sentence of the present study were different from each other, so that a different symbol would have to be retrieved on each recoding trial. For example, to recode not hot, the representation of cold must be retrieved from semantic memory. This retrieval time may have inflated the latencies for the predicate negatives.

\section{Recall}

The verification latencies indicated that, at the time of comprehension, predicate negative sentences are often recoded into equivalent affirmative forms. By contrast, denials were represented in a negative form at the time of comprehension. This analysis of the verification results predicts that predicate negatives should later be recalled in an equivalent affirmative form more often than denials. As the second column of Table 2 shows, this prediction was confirmed. Predicate negatives were recalled in an equivalent affirmative form over four times as often as denials, $F^{\prime}(1.156)=9.86, p<.01$.

Additional evidence of recoding is provided by the frequency of recall of the antonym of the originally presented adjective, regardless of whether the response form was affirmative, predicate negative, or denial. A predicate negative sentence that has been recoded contains an element that is antonymous to the originally presented adjective; therefore, people may recall the antonym of the adjective they had actually seen in the predicate negative sentence. For the denial sentences, which were not recoded, this type of erroneous recall should be much less frequent. As Table 2 shows, the tendency to report the antonymous adjective was over three times greater for predicate negatives $(.15)$ than denials $(.04), F^{\prime}(1,168)=12.73$, $\mathrm{p}<.01$. This result is consistent with the finding that during verification, predicate negative sentences were often recoded, but denials were not.

The pattern of recall for true and false predicate negatives indicated that constructive processes (James, Thompson, \& Baldwin, 1973) were operating at the time of recall. In particular, there may be a reconstructive bias to respond with the adjective (i.e., the point on the adjectival dimension) that is typically

Table 2

Distribution of Recall Responses (Proportions Based on 180 Observations)

\begin{tabular}{|c|c|c|c|c|c|c|c|c|c|c|}
\hline \multirow{3}{*}{$\begin{array}{l}\text { Stimulus } \\
\text { Sentence Type }\end{array}$} & & \multicolumn{3}{|c|}{ Affirmative } & \multicolumn{3}{|c|}{$\begin{array}{c}\text { Response Sentence Type } \\
\text { Predicate Negative }\end{array}$} & \multicolumn{3}{|c|}{ Denial } \\
\hline & & \multicolumn{3}{|c|}{ Adjective Recalled } & \multicolumn{3}{|c|}{ Adjective Recalled } & \multicolumn{3}{|c|}{ Adjective Recalled } \\
\hline & & Verbatim & Antonym & Error & Verbatim & Antonym & Error & Verbatim & Antonym & Error \\
\hline \multirow[t]{2}{*}{ Affirmative } & True & .62 & .03 & .22 & .02 & .01 & .01 & .05 & .01 & .03 \\
\hline & & & & & & & & & & \\
\hline Predicate & True & .21 & .14 & .29 & .13 & .02 & .03 & .10 & .02 & .05 \\
\hline Negative & False & .31 & .07 & .28 & .13 & .02 & .01 & .11 & .02 & .02 \\
\hline \multirow[b]{2}{*}{ Denial } & True & .37 & .03 & .32 & .06 & .01 & .01 & .18 & .01 & .01 \\
\hline & False & .38 & .02 & .31 & .09 & .01 & .02 & .16 & .01 & .00 \\
\hline
\end{tabular}


associated with that noun. For example, to reconstruct the sentence It is true that a fire isn't cold when only the truth value and the adjectival dimension can be retrieved, the reconstructive bias might select the adjective hot, which is usually associated with fire. This bias would produce the sentence It is true that a fire is hot, i.e., the equivalent affirmative form. By contrast, when the predicate negative sentence is false, the reconstructive bias would enhance the reporting of the adjective that had actually been presented. Consistent with this proposal. the results show that false predicate negatives were recalled in a recoded form (i.e., an affirmative sentence with the antonymous adjective) about half as often as true predicate negatives $(.07 \mathrm{vs}$. .14). Furthermore, false predicate negatives were recalled in a nonequivalent affirmative form (i.e., with the original adjective) more often than true predicate negatives $(.31$ vs. .21$)$. The difference in the recall pattern between true and false predicate negatives is apparently due to the reconstructive bias operating at the time of recall.

The verification latencies indicated that there were individual differences among the subjects in how they verified predicate negatives. The 18 subjects fell into three groups. Eight of the subjects were recoders, as indicated by the shorter latencies (by at least $150 \mathrm{msec}$ ) for true predicate negatives than for false predicate negatives. For seven other subjects, latencies were longer by at least $150 \mathrm{msec}$ for true predicate negatives than for false ones, indicating that the latter group was not recoding. Finally, three subjects fell into neither group, with the difference between true and false predicate negatives falling below $44 \mathrm{msec}$ for each of the three subjects. These last three subjects presumably alternated between recoding and regular processing, resulting in equal latencies for true and false predicate negatives. For all three groups of subjects, false denials were verified faster than true ones, indicating that denials were never recoded. The recall data did not reveal individual differences corresponding to those found in verification. Predicate negatives were recalled in the equivalent affirmative form about as often by the recoders $(9 \%$ of the time) as by the nonrecoders (11\%). A similar pattern was obtained in the recall of the adjective, irrespective of the recalled syntactic form. This suggests that some of the subjects who did not recode predicate negatives during verification did recode them at the time of recall. As expected, neither group recoded denials at the time of recall or during comprehension.

The analyses reported above were on the $0-1$ frequencies of the various types of responses. These analyses allowed us to examine the generality of the results over both random factors-propositions and people. However, other analyses that collapsed over one or the other population and applied an arcsin transformation to the resulting proportions yielded similar results. Also, an analysis that considered only those sentences that had been correctly verified yielded similar results. And finally, while the reported analyses gave subjects credit only for verbatim recall of the adjective, another more lenient scoring procedure credited synonyms, e.g., big for large. It, too, produced similar results. There were a few cases where sentences were recalled as double negatives $(.02, .04$, and .03 for affirmatives, predicate negatives, and denials, respectively). These do not appear in Table 2.

The performance data from the verification task and the recall task indicate that the two types of negative sentences are treated differently from each other at both stages. Denials are not recoded at the time of comprehension, nor are they reported in a recoded form at the time of recall. Nor is there much tendency to report the antonymous adjective at the time of recall. The recall of denials does show a strong tendency to delete the negation, and only report the adjective that occurred. Subjects were often unable to recall that the sentence had been negative, and so they reported it as an affirmative. This type of response was the most frequent (about $37 \%$ ) and indicates that the subjects were able to recall the correct adjective equally well regardless of whether the denial was true or false. In some sense, the affirmative report may have been a default option, which the subjects used when they could not retrieve the polarity or the truth value of the sentence.

Predicate negative sentences tend to be recoded at the time of comprehension and at the time of recall. The recoding tendency in recall was stronger for the true predicate negatives, indicating that a reconstructive bias may have encouraged the recall of an associated adjective. The subjects who recoded predicate negatives during comprehension may simply have recalled the recoded representation. The other subjects must have recoded the predicate negatives during some stage after verification.

One issue that arises in this and in previous studies is why predicate negatives are sometimes spontaneously recoded at the time of comprehension, but denials never are spontaneously recoded. One conjecture is that recoding occurs only when the negative marker is immediately adjacent to an affirmative proposition in the internal representation. That is, the negation is recoded only if it is right next to the core proposition. For predicate negatives, the 
negation immediately embeds the inner proposition, and those sentences are sometimes recoded. However, for denials, the negation applies to an entire proposition which has its own affirmative marker. In this case, recoding does not occur. This type of explanation is consistent with the verification latencies. It may also apply to the memory results, in that recoding could occur at the time of recall if the sentence had been a predicate negative.

By tracing the processing history of these two types of negatives, we have shown that there is a correlation between their representations during comprehension and subsequent recall. Only those types of negatives that are recoded during comprehension tend to be recalled in a recoded form. However, it would be incorrect to conclude that recall performance can be entirely accounted for in terms of comprehension processes. This point is clearly demonstrated by those subjects who did not recode predicate negatives during comprehension, but did recode them at the time of recall. Nevertheless, the results of this study indicate that recoding of negative sentences is not purely a memory phenomenon, but rather it is a process that also occurs at previous stages.

With the knowledge of how the sentences were processed in the present task, we can return to Fillenbaum's (1966) study and discuss the source of his effect. He found that negative sentences like The door isn't closed tend to be recalled as The door is open. The usual explanation for this result is that the format in which the information is stored retains only the gist of a sentence. When it comes time to recall the sentence, the gist is retrieved, and expressed in the syntactically simplest way. But this is only part of the story. The present analysis indicates that complex sentences are often recoded into a simpler form during the act of comprehension. Later, during recall of the sentence, the recoded representation may be retrieved. This explanation indicates that the "memory for gist" effect may be due largely to a "comprehension of gist" process.

\section{REFERENCE NOTE}

1. Young, R., \& Chase, W. G. Additive stages in the comparison of sentences and pictures. Paper presented at Midwestern Psychological Association meetings, April 1971.

\section{REFERENCES}

Carpenter. P. A. Extracting information from counterfactual clauses. Journal of Verbal Learning and Verbal Behavior, $1973,12,512-521$.

Carpenter, P. A., \& Just, M. A. Sentence comprehension: A psycholinguistic processing model of verification. Psychological Review, 1975, 82, 45-73.

CLARK, H. H. The language-as-fixed-effect fallacy: A critique of language statistics in psychological research. Journal of Verbal Leaming and Verbal Behavior, 1973, 12, 335-359.

Clark, H. H., \& Chase, W. G. On the process of comparing sentences against pictures. Cognitive Psychology, 1972, 3. 472-517.

Fillenbaum, S. Memory for gist: Some relevant variables. Language and Speech. 1966, 9. 217-227.

FilleNBAUM, S. Syntactic factors in memory. Janua Linguarum, Series Minor. The Hague: Mouton, 1973.

JACKENDOFF, R. S. An interpretative theory of negation. Foundations of Language, 1969, 5, 218-241.

James, C. T., Thompson, J. G., \& Baldwin, J. M. The reconstructive process in sentence memory. Journal of Verbal Leaming and Verbal Behavior, 1973, 12, 51-63.

Just. M. A., \& Carpenter. P. A. Comparative studies of comprehension: An investigation of Chinese. Norwegian, and English. Memory \& Cognition, 1975, 3. 465-473.

KintsCH, W. Notes on the structure of semantic memory. In E. Tulving \& W. Donaldson (Eds.), Organization of memony. New York: Academic Press. 1972.

KLImA, E. S. Negation in English. In J. A. Fodor \& J. J. Katz (Eds.). The structure of language. Englewood Cliffs, N.J: Prentice-Hall, 1964.

Trabasso. T. Mental operations in language comprehension. In J. B. Carroll \& R. O. Freedle (Eds.), Language comprehension and the acquisition of knowledge. Washington. D.C: Winston, 1972.

WAson. P. C. Response to affirmative and negative binary statements. British Journal of Psychology, 1961, 52, 133-142.

WINER. B. J. Statistical principles in experimental design. New York: McGraw-Hill, 1971.

(Received for publication August 18, 1975; revision received October 15,1975 .) 